Proceedings of the 6th Polish Symposium of Physics in Economy and Social Sciences (FENS2012), Gdańsk, Poland

\title{
Subsequent Movements' Proportions of Share Prices Included in the WIG over Recent Years
}

\section{A. SzMAgLińsKi}

Institute of Physics (PK), Cracow University of Technology, Podchorążych 1, 30-084 Kraków, Poland

A large amount of stock prices intraday data allow us to create a summary of subsequent movements' proportions of the collected share prices in the form of histogram. We have created two kinds of histograms: one for proportions of subsequent increasing and decreasing price movements and the second for proportions of subsequent price movements in the same direction. We have also created the same kinds of histograms for duration of price movements. All the histograms quite well fit the gamma probability distribution. The distribution coefficients' values $\nu$ and $\lambda$ for price are above 1, for time are below 1. Some proportions of price movements occur more frequently than others, creating peaks on the graph. Similar regularity occurs for the time factor. This property is often used in trading.

DOI: $10.12693 /$ APhysPolA.123.621

PACS: 05.45.Df, 05.45.Tp, 89.65.Gh

\section{Introduction}

One of the main applications of technical analysis is the estimation of price movement probability in chosen direction. In this paper we will focus on two kinds of subsequent movements' proportions in price and time. Firstly on the proportion of correction to impulse for increasing and decreasing price movements (the impulse may increase or decrease). Secondly on the proportion of subsequent impulses (the price movements have the same directions). For the research we apply the collection of shares included in Warszawski Indeks Giełdowy (WIG) quoted in the Warsaw Stock Exchange from October 2005 until April 2012.

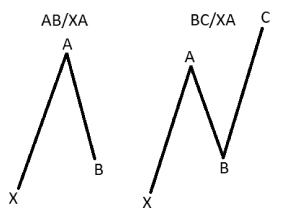

Fig. 1. Two kinds of measuring the price movement proportions in price and time. The first proportion of correction to impulse and the second proportion of subsequent impulses.

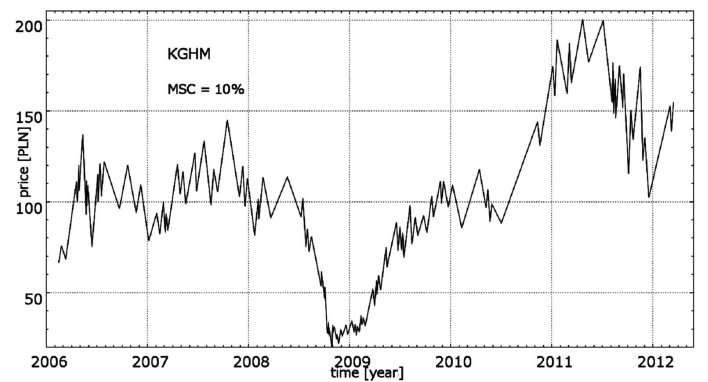

Fig. 2. Price-time series for shares of KGHM with ignored price movements of less than the minimum size of correction $=10 \%$.

A great number of impulse and correction proportions allow us to set them in histograms. Here we have two kinds of proportions as shown in Fig. 1. The idea of how to simplify the price movements is based on the Point \& Figure charts as described in [1] and Kagi charts, which were developed in Japan [2]. The price movements in the opposite direction, which are less than the fixed percentage (called here minimum size of correction MSC), are ignored. In most examples presented here MSC equals $10 \%$. So finally we have price-time series composed of turning points. Additionally we introduce two conditions, so as to avoid price movements corresponding to the random fluctuations (for example realization of best buy and sell offers). The first condition: when the share price is less than 1 PLN, every percent of MSC corresponds to 0.01 PLN. The second condition: MSC > 0.50 PLN. The example price-time series for shares of KGHM is printed in Fig. 2.

\section{The correction-impulse price movement proportions}

The most simple case corresponds to subsequent impulse and correction. The proportions correction/impulse are set in histogram. Every case of such a proportion is placed in a narrow range of thickness 0.01 , forming a part of the histogram. The same procedure is used for the duration of this price movements. In Fig. 3 there are shown two histograms for price proportions and time proportions. These histograms are well fitted with gamma probability distribution functions

$$
\Gamma_{\nu, \lambda}(x)=C x^{\nu-1} \mathrm{e}^{-\lambda x} .
$$

The coefficients of the fitted gamma probability distribution functions are placed in Table. The gamma probability distribution in more complicated form was applied for the investment horizon distribution [3]. For particular asset the investment horizon is the smallest time interval needed for the asset to cross a fixed return level. In the paper mentioned above calculations were performed on the historic daily closure prices of the Dow Jones Industrial Average. For instance with the return level of $5 \%$, the maximum of gamma probability distribution function is at the optimal investment horizon with value of approximately 15 trading days. The gain-loss 
asymmetry was discussed in [4], where negative return levels were considered. In paper [5] intraday foreign exchange time series were analyzed with waiting physical times and tick times, needed to obtain a certain increase (decrease) in price. Finally, the investment horizon approach was used to study characteristics of the Polish Pioneer mutual funds [6]. Though the similar gamma distribution functions describe the investment horizon distribution and the subsequent price movement duration proportions, there is no direct connection between investment horizon and price movement duration or its proportions. This is a different kind of analyzing time in the price movements.

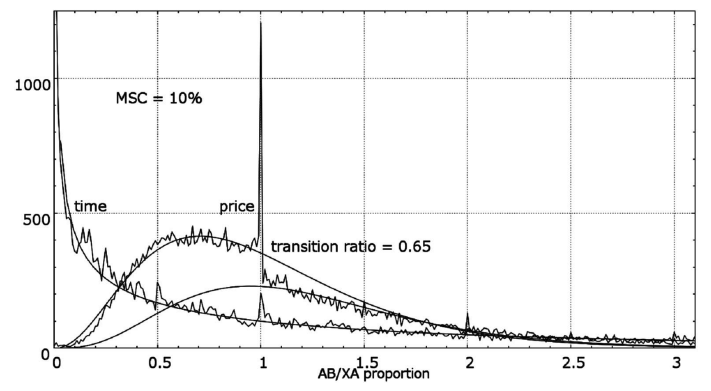

Fig. 3. Two histograms for price and time correction/ impulse proportions with gamma probability distribution functions fitted. In the histogram of price at 1 we have the sudden drop in probability of completing price movement. The probability rapidly decreases to $65 \%$ of the previous one while crossing 1 .

TABLE

The coefficients of fitted gamma probability distribution functions for histograms of price movements proportions with $\mathrm{MSC}=10 \%$.

\begin{tabular}{c|c|c|c|c|c}
\hline \hline Proportions & Name & $C$ & $\nu$ & $\lambda$ & $\Gamma(1)$ \\
\hline \multirow{3}{*}{ correction-impulse } & price L & 10742 & 3.417 & 3.418 & 352.09 \\
& price R & 8578 & 4.443 & 3.626 & 228.37 \\
& time & 143.22 & 0.512 & 0.364 & 99.52 \\
\hline \multirow{2}{*}{ impulse-impulse } & price & 10259 & 3.395 & 3.443 & 327.97 \\
& time & 151.62 & 0.543 & 0.394 & 102.25
\end{tabular}

Assuming that, for every proportion of neighboring price motions, in magnitude or in duration in time, exists inverse proportion, the number of proportions in the range $\langle 0.51 ; 0.99\rangle$ should be close to the number of proportions in the range $\langle 1.01 ; 1.98\rangle$. In practice, for proportions of price movements' duration it works better than for proportions of price movements at its magnitude. But this effect is visible in the distribution form. The proportions of price movements $0.51<A B / X A<0.99$ or $0.51<B C / X A<0.99$ are more often than others.

In the histogram of price proportions there is sudden drop in probability distribution, corresponding to the breaking of the nearest turning point resistance. The expectations of market participants change after breaking of the resistance. The probability of price movement completion rapidly decreases when the second price movement becomes greater than the first one. This de- crease is estimated by fitting two gamma probability distribution functions for proportions: one greater than 1 and the second lesser than 1 . This ratio of transition from one probability distribution to the other for $\mathrm{MSC}=10 \%$ equals $65 \%$, and for $\mathrm{MSC}=5 \%$ equals $57 \%$ and this rapid decrease is stronger in general for the lesser magnitude of price movements. The effect of resistance breaking is widely known among the investors and the change in probability of price movement's continuation in the same direction here is given quantitatively. Prior resistance becomes the support area and this can be used as one of possible investment strategy components. One can expect the continuation of price movement. When the increase in price is expected, the strategy gives a buy signal with stop-loss below the support area (for instance a few transaction units below the broken turning point).

For some values of price and time proportions there are peaks. They correspond to proportions in price and time with greater probability of setting a turning point. The special proportion is the equality of impulse and correction. This proportion is much greater than the background. The probability of trend changing is much greater than in any other nearing price (for the price histogram) or time (in regard to the time histogram). The peak corresponding to the equality of duration of impulse and correction is much more diffused. Such a behavior of price is frequently used in technical analysis, and recently the behavior of time factor is also used. The peaks has its investment application as an accessory condition. For instance when the price movement has changed direction at the peak area (it means with greater probability of changing direction) and the price is still not far from this area. The effect intensifies with the overlapping peaks originating from the comparison with other turning points of different price scales. The same applies to the price movement duration. Among some investors it is called the "geometry of price and time" and is used as an accessory investment tool together with, for instance, candlestick patterns.



Fig. 4. The histogram of price movement of impulse and correction, which are the same in value. The values of price movements are placed in a logarithmic scale.

For the main peak at 1 the price movement of impulse and correction, which are the same in value with accuracy of $0.5 \%$, are placed in the histogram in Fig. 4 . The price is presented in a logarithmic scale and decreases in number of proportion cases, exceeding 100 PLN. 
In a similar way the peak for duration of impulse and correction at 1 is presented in Fig. 5. The duration of impulse and the duration of correction are the same with accuracy of $0.5 \%$ in this case. Some price movements last one day, but most of them last several days till tens of days.

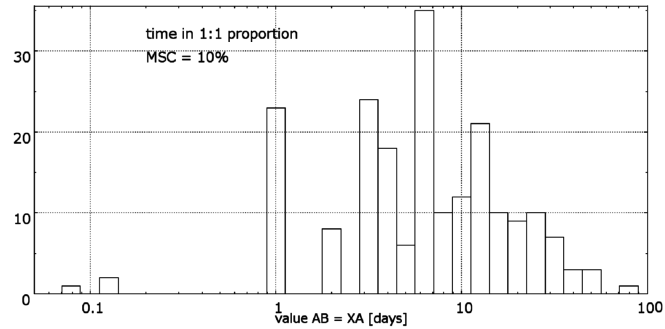

Fig. 5. The histogram of price movement duration of impulse and correction, which are the same in value.

The values of time are placed in a logarithmic scale.

Further investigations of the peak in 1 in price and time proportions do not show the meaningful cross correlations. There is no significant correlations both in correction-impulse proportions and impulse-impulse proportions.

\section{The impulse-impulse price movements proportions}

The proportions of subsequent impulses have similar properties to the correction-impulse proportions with one exception. The sudden drop in probability distribution corresponding to the breaking of turning point resistance is not noticeable (Fig. 6). There is one gamma probability distribution function for the histogram of price proportions and one for the time proportions. The coefficients of these fitted gamma functions are placed in Table.

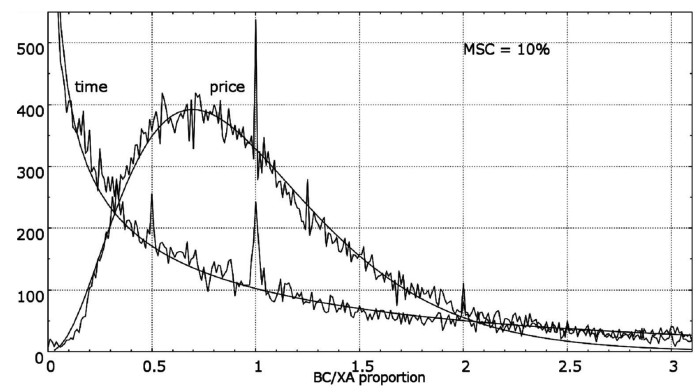

Fig. 6. The histogram of subsequent impulses' price movement proportions and duration of impulses proportions.

The main peaks are also in 1 . When we subtract the fitted gamma function from the histogram we can clearly see the peaks in price (Fig. 7) and time (Fig. 8). In price there are peaks corresponding to the multiple of the fraction of numbers 2 and 3 . In time we have also small peaks corresponding to the division by 7 , which may originate from the 7 days in a week.

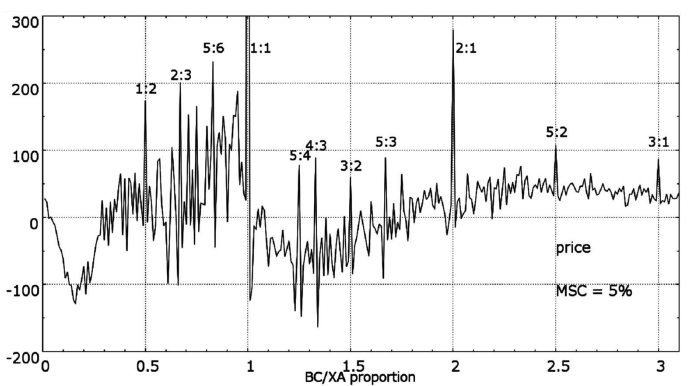

Fig. 7. Subtraction of the fitted gamma probability distribution function from the price proportion histogram. We can clearly see the peaks in price, which correspond to the proportions of small integer numbers.

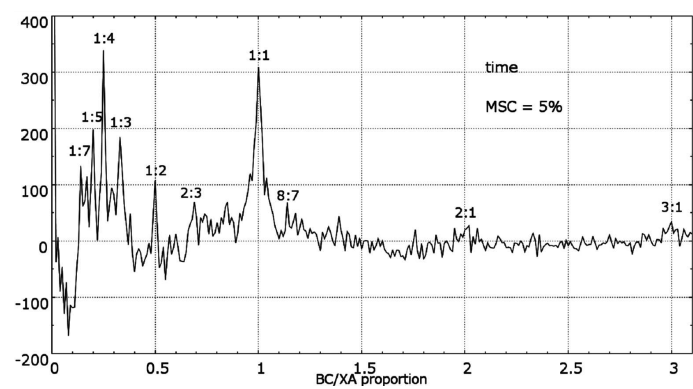

Fig. 8. Subtraction of the fitted gamma probability distribution function from the duration of impulses proportions histogram. We can clearly see the peaks in time, which correspond to the proportions of small integer numbers.

\section{Summary}

In this paper we have demonstrated that the shares have a tendency of repeatability of price movements both in value of price movements and in these movements' time duration. For this repeatability is responsible for the peak in 1 in the histograms of price and time. The effect of breaking the turning point resistance is presented quantitatively. This breaking of resistance makes the probability of price movement completion less by tens of percents. The greater scale of price movement the lesser decrease of the probability.

\section{References}

[1] T.J. Dorsey, Point and Figure Charting. The Essential Application for Forecasting and Tracking Market Prices, Wiley, 1995.

[2] S. Nison, Beyond Candlesticks. New Japanese Charting Techniques Revealed, Wiley, 1994.

[3] I. Simonsen, M.H. Jensen, A. Johansen, Eur. Phys. J. 27, 583 (2002).

[4] M.H. Jensen, A. Johansen, I. Simonsen, Int. J. Mod. Phys. B 17, 4003 (2003).

[5] M.H. Jensen, A. Johansen, F. Petroni, I. Simonsen, Physica A 340, 678 (2004).

[6] M. Grudziecki, E. Gnatowska, K. Karpio, A. Orłowski, M. Załuska-Kotur, Acta Phys. Pol. A 114, 569 (2008). 